\title{
Stone Duality for Markov Processes
}

\author{
Dexter Kozen ${ }^{1} \quad$ Kim G. Larsen ${ }^{2} \quad$ Radu Mardare ${ }^{2}$ \\ Prakash Panangaden ${ }^{3}$ \\ ${ }^{1}$ Computer Science Department, Cornell University, Ithaca, New York, USA \\ ${ }^{2}$ Department of Computer Science, University of Aalborg, Aalborg, Denmark \\ ${ }^{3}$ School of Computer Science, McGill University, Montreal, Canada
}

March 14, 2013

\begin{abstract}
We define Aumann algebras, an algebraic analog of probabilistic modal logic. An Aumann algebra consists of a Boolean algebra with operators modeling probabilistic transitions. We prove that countable Aumann algebras and countably-generated continuous-space Markov processes are dual in the sense of Stone. Our results subsume existing results on completeness of probabilistic modal logics for Markov processes.
\end{abstract}

\section{Introduction}

The Stone representation theorem [1] is a recognized landmarks of mathematics. The theorem states that every (abstract) Boolean algebra is isomorphic to a Boolean algebra of sets. In fact, Stone proved much more: that Boolean algebras are dual to a certain category of topological spaces known as Stone spaces. See Johnstone [2] for a detailed and eloquent introduction to this theorem and its ramifications, as well as an account of several other related dualities, like Gelfand duality, that appear in mathematics.

Formally, Stone duality states that the category of Boolean algebras and Boolean algebra homomorphisms and the category of Stone spaces and continuous maps are contravariantly equivalent. Jonsson and Tarski [3] extended this to modal logic and showed a duality between Boolean algebras 
with additional operators and certain topological spaces with additional operators.

Stone duality embodies completeness theorems, but goes far beyond them. Proofs of completeness typically work by constructing an instance of a model from maximal consistent sets of formulas. Stone duality works in the same way, but gives a correspondence not just for syntactically generated algebras, but for any suitable algebra. This includes both smaller algebras, which could be finite and generate finite structures, or larger algebras, which could be uncountable, thus not syntactically generated. Furthermore, homomorphisms of algebras give rise to maps between the corresponding structures in the opposite direction. Thus mathematical arguments can be transferred in both directions.

Stone-type dualities are now recognized as being ubiquitous in computer science. Plotkin [4] and Smyth [5] emphasized that the duality between state-transformer semantics and predicate-transformer semantics is an instance of a Stone-type duality. Kozen [6] discovered such a duality for probabilistic transition systems. Abramsky [7] identified dualities in domain and concurrency theory. Recently several authors (e.g. [8,9]) have emphasized the duality between logics and transition systems from a coalgebraic perspective. Mislove et al. [10] found a duality between labeled Markov processes and $C^{*}$-algebras based on the closely related classical Gelfand duality.

For Markov processes, the natural logic is a simple modal logic in which bounds on probabilities enter into the modalities. This logic can be stripped down to a very spartan core-just the modalities and finite conjunctionand still characterize bisimulation for labeled Markov processes [11,12]. It is therefore tempting to understand this logic algebraically in the same way that Boolean algebras capture propositional reasoning and the JonssonTarski results give duality for algebras arising from modal logics.

In this paper, we develop a Stone-type duality for continuous-space probabilistic transitions systems and a certain kind of algebra that we have named Aumann algebras. These are Boolean algebras with operators that behave like probabilistic modalities. Recent papers [13-15] have established completeness theorems and finite model theorems for similar logics. Thus it seemed ripe to attempt to capture these logics algebraically and explore duality theory. This is what we do in this paper.

A comparison with related work appears in $\S 7$. We note here that we go beyond existing completeness results [13-15] in a number of ways. Follow- 
ing the pioneering work of Goldblatt [14], the strong completeness theorems use a powerful infinitary axiom scheme called the countably additivity rule in [14]. This rule has an uncountable set of instances. Moreover, for strong completeness, one needs to postulate Lindenbaum's lemma, which for the aforementioned logics has not been proven. In this paper we use the Rasiowa-Sikorski lemma [16] to finesse Goldblatt's axiom and prove Lindenbaum's lemma.

Our key results are:

- a description of a new class of algebras that capture in algebraic form the probabilistic modal logics used for continuous-state Markov processes;

- a version of duality for countable algebras and a certain class of countablygenerated Markov processes; and

- a complete axiomatization that does not involve infinitary axiom schemes with uncountably many instances and avoids postulating Lindenbaum's lemma as a meta-axioms.

The duality is represented in the diagram below. Here SMP stands for Stone Markov processes and AA for countable Aumann algebras. The formal definitions are given in $\S \S 3-4$.

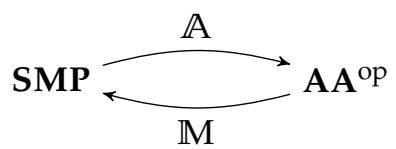

A general Markov process gives rise to a countable Aumann algebra but a countable Aumann algebra gives rise to a Stone Markov process. However, starting from a Markov process $\mathcal{M}$, constructing the corresponding Aumann algebra $\mathcal{A}$, then constructing the corresponding Stone Markov process corresponding $\mathcal{A}$, one obtains a Markov process bisimilar to $\mathcal{M}$.

\subsection{A Summary for Experts}

The duality theorem proved in this paper has some novel features that distinguish it from many others that have appeared in the literature. 
First and foremost, we have minimized the use of infinitary axioms [17] by using the Rasiowa-Sikorski lemma (whose proof uses the Baire category theorem) in the following way. In going from the algebra to the dual Markov process, we look at ultrafilters that do not respect the infinitary axioms of Aumann algebras. We call these bad ultrafilters. We show that these form a meager set (in the sense of the Baire category theorem) and can be removed without affecting the transition probabilities that we are trying to define. Countability is essential here. In order to show that we do not affect the algebra of clopen sets by doing this, we introduce a distinguished base of clopen sets in the definition of Markov process, which has to satisfy some conditions. We show that this forms an Aumann algebra. We are able to go from a Markov process to an Aumann algebra by using this distinguished base. Morphisms of Markov processes are required to preserve distinguished base elements backwards; that is, if $f: \mathcal{M} \rightarrow \mathcal{N}$ and $A \in \mathcal{A}_{\mathcal{N}}$, then $f^{-1}(A) \in \mathcal{A}_{\mathcal{M}}$. Thus we get Boolean algebra homomorphisms in the dual for free.

Removing bad points has the effect of destroying compactness of the resulting topological space. We introduce a new concept called saturation that takes the place of compactness. The idea is that a saturated model has all the good ultrafilters. The Stone dual of an Aumann algebra is saturated, because it is constructed that way. However, it is possible to have a Markov process that is unsaturated but still represents the same algebra. For example, we removed bad points and could, in principle, remove a few more; as long as the remaining points are still dense, we have not changed the algebra. One can saturate a model by a process akin to compactification. We explicitly describe how to do this below.

\section{Background}

Let $\mathbb{Q}_{0}=\mathbb{Q} \cap[0,1]$ and $\mathbb{R}^{+}=\mathbb{R} \cap[0, \infty)$.

\subsection{Measurable Spaces and Measures}

In this section we introduce a few concepts and results from measure theory that we will find useful. All of these results are well known. For proofs and related results, we refer the reader to [18] or [19].

Let $M$ be an arbitrary nonempty set. 
- A field (of sets) over $M$ is a Boolean algebra of subsets of $M$ under the usual set-theoretic Boolean operations.

- A $\sigma$-algebra over $M$ is a field of sets over $M$ closed under countable union. The tuple $(M, \Sigma)$ is called a measurable space and the elements of $\Sigma$ measurable sets.

Given two measurable spaces $(M, \Sigma)$ and $(N, \Omega)$, a function $f: M \rightarrow N$ is measurable if $f^{-1}(T) \in \Sigma$ for all $T \in \Omega$. We use $\llbracket M \rightarrow N \rrbracket$ to denote the family of measurable functions from $(M, \Sigma)$ to $(N, \Omega)$.

If $\Omega \subseteq 2^{M}$, the $\sigma$-algebra generated by $\Omega$, denoted $\Omega^{\sigma}$, is the smallest $\sigma$-algebra containing $\Omega$.

A nonnegative real-valued set function $\mu$ is finitely additive if $\mu(A \cup B)=$ $\mu(A)+\mu(B)$ whenever $A \cap B=\varnothing$. We say that $\mu$ is countably subadditive if $\mu\left(\bigcup_{i} A_{i}\right) \leq \sum_{i} \mu\left(A_{i}\right)$ for a countable family of measurable sets, and we say that $\mu$ is countably additive if $\mu\left(\cup_{i} A_{i}\right)=\sum_{i} \mu\left(A_{i}\right)$ for a countable pairwisedisjoint family of measurable sets.

Finite additivity implies monotonicity and countable additivity implies continuity properties as stated in the theorems below.

Theorem 1. Let $\mathcal{F} \subseteq 2^{M}$ be a field of sets. If $\mu: \mathcal{F} \rightarrow \mathbb{R}^{+}$is finitely additive, then

(i) $\mu(\varnothing)=0$;

(ii) $\mu$ is monotone: if $A \subseteq B$ then $\mu(A) \leq \mu(B)$; and

(iii) if $A \subseteq B$ then $\mu(B \backslash A)=\mu(B)-\mu(A)$.

The next theorem provides five equivalent properties that a finitely additive function over a field of sets can have.

Theorem 2. Let $\mathcal{F} \subseteq 2^{M}$ be a field of sets. Let $\mu: \mathcal{F} \rightarrow \mathbb{R}^{+}$be finitely additive. The following are equivalent:

(i) $\mu$ is countably subadditive: for any countable collection $A_{i}$ such that $\bigcup_{i} A_{i} \in$ $\mathcal{F}$,

$$
\mu\left(\bigcup_{i} A_{i}\right) \leq \sum_{i} \mu\left(A_{i}\right) ;
$$


(ii) $\mu$ is countably additive: for any countable collection $A_{i}$ such that $\bigcup_{i} A_{i} \in \mathcal{F}$ and the $A_{i}$ are pairwise disjoint,

$$
\mu\left(\bigcup_{i} A_{i}\right)=\sum_{i} \mu\left(A_{i}\right)
$$

(iii) $\mu$ is $\omega$-continuous from below: for any countable chain $A_{0} \subseteq A_{1} \subseteq \cdots$ such that $\bigcup_{i} A_{i} \in \mathcal{F}$,

$$
\mu\left(\bigcup_{i} A_{i}\right)=\sup _{i} \mu\left(A_{i}\right)
$$

(iv) $\mu$ is $\omega$-continuous from above: for any countable chain $A_{0} \supseteq A_{1} \supseteq \ldots$ such that $\bigcap_{i} A_{i} \in \mathcal{F}$,

$$
\mu\left(\bigcap_{i} A_{i}\right)=\inf _{i} \mu\left(A_{i}\right)
$$

(v) $\mu$ is $\omega$-continuous from above at $\varnothing$ : for any countable chain $A_{0} \supseteq A_{1} \supseteq \cdots$ such that $\bigcap_{i} A_{i}=\varnothing$,

$$
\mu\left(\bigcap_{i} A_{i}\right)=0 .
$$

A measure on a measurable space $\mathcal{M}=(M, \Sigma)$ is a countably additive set function $\mu: \Sigma \rightarrow \mathbb{R}^{+}$. Observe that all measures satisfy the five equivalent properties stated in Theorem 2.

The next theorem is a key tool in our constructions.

Theorem 3 (Theorem 11.3 of [18]). Let $\mathcal{F} \subseteq 2^{M}$ be a field of sets. Let $\mu: \mathcal{F} \rightarrow$ $\mathbb{R}^{+}$be finitely additive and countably subadditive. Then $\mu$ extends uniquely to a measure on $\mathcal{F}^{\sigma}$.

Proof sketch. This argument is known as the Carathéodory construction. For $B \in 2^{M}$, define

$$
\mu^{*}(B)=\inf _{\substack{B \subseteq \cup \mathcal{C} \\ \mathcal{C} \text { countable }}} \sup _{F \subseteq \mathcal{C} \text { finite }} \mu(\bigcup F)
$$

where the infimum ranges over countable $\mathcal{F}$-covers $\mathcal{C}$ of $B$. The map $\mu^{*}$ is called an outer measure of $\mu$. One can show that $\mu^{*}$ satisfies the following properties: 
- $\mu^{*}$ and $\mu$ agree on $\mathcal{F}$;

- $\mu$ is monotone;

- $\mu$ is countably subadditive.

Now define a set $B$ to be measurable with respect to $\mu^{*}$ if for all $A \in 2^{M}$,

$$
\mu^{*}(A)=\mu^{*}(A \cap B)+\mu^{*}(A \backslash B) .
$$

One now shows that the set of measurable sets is a $\sigma$-algebra, therefore contains $\mathcal{F}^{\sigma}$, and all properties of measures are satisfied.

A measure is a probability measure if in addition $\mu(M)=1$. We use $\Delta(M, \Sigma)$ to denote the class of probability measures on $(M, \Sigma)$. We can view $\Delta(M, \Sigma)$ as a measurable space by considering the $\sigma$-algebra generated by the sets $\{\mu \in \Delta(M, \Sigma) \mid \mu(S) \geq r\}$ for $S \in \Sigma$ and $r \in[0,1]$. This is the least $\sigma$-algebra on $\Delta(M, \Sigma)$ such that all maps $\mu \mapsto \mu(S): \Delta(M, \Sigma) \rightarrow[0,1]$ for $S \in \Sigma$ are measurable, where the real interval $[0,1]$ is endowed with the $\sigma$-algebra generated by all rational intervals.

\subsection{Analytic Spaces}

It turns out that one obtains a much richer theory when one combines measure theory and topology; in fact a standard restriction is to consider so called Polish spaces. We will work with a broader class called analytic spaces. We define these and related concepts here.

Every topological space has a natural $\sigma$-algebra associated with it, namely the one generated by the open sets. This is called the Borel algebra of the space, and the measurable sets are called Borel sets.

Recall that a topological space is said to be separable if it contains a countable dense subset and second countable if its topology has a countable base. Second countability implies separability, but not vice versa in general; however, the two concepts coincide for metric spaces.

A Polish space is the topological space underlying a complete separable metric space.

An analytic space is a continuous image of a Polish space. More precisely, if $X$ and $Y$ are Polish spaces and $f: X \rightarrow Y$ is continuous, then the image $f(X)$ is an analytic space. 
Remarkably, one does not get a broader class by allowing $f$ to be merely measurable instead of continuous and by taking the image of a Borel subset of $X$ instead of $X$. That is, the measurable image of any Borel subset of a Polish space is analytic.

Analytic spaces enjoy remarkable properties that were crucial in proving the logical characterization of bisimulation $[12,20]$. We note that the completeness theorems proved in $[13,21,22]$ were established for Markov processes defined on analytic spaces.

\subsection{The Baire Category Theorem}

The Baire category theorem is a topological result with important applications in logic. It can be used to prove the Rasiowa-Sikorski lemma [16] that is central for our paper.

A subset $D$ of a topological space $X$ is dense if its closure $\bar{D}$ is all of $X$. Equivalently, a dense set is one intersecting every nonempty open set. A set $N \subseteq X$ is nowhere dense if every nonempty open set contains a nonempty open subset disjoint from $N$. A set is said to be of the first category or meager if it is a countable union of nowhere dense sets. The term "meager" is meant to suggest that these sets are small in a topological sense. A basic fact that we use is that the boundary of an open set is nowhere dense.

A Baire space is one in which the intersection of countably many dense open sets is dense. It follows from these definitions that the complement of a first category set is dense in any Baire space. Baire originally proved that the real line is a Baire space. More generally, every Polish space is Baire and every locally compact Hausdorff space is Baire. For us, the relevant version is the following special case: every compact Hausdorff space is Baire.

Definition 4. Let $\mathcal{B}$ be a Boolean algebra and let $T \subseteq \mathcal{B}$ such that $T$ has a greatest lower bound $\wedge T$ in $\mathcal{B}$. An ultrafilter (maximal filter) $U$ is said to respect $T$ if $T \subseteq U$ implies that $\wedge T \in U$.

If $\mathcal{T}$ is a family of subsets of $\mathcal{B}$, we say that an ultrafilter $U$ respects $\mathcal{T}$ if it respects every member of $\mathcal{T}$.

Theorem 5 (Rasiowa-Sikorski lemma [16]). For any Boolean algebra $\mathcal{B}$ and any countable family $\mathcal{T}$ of subsets of $\mathcal{B}$, each member of which has a meet in $\mathcal{B}$, and for any nonzero $x \in \mathcal{B}$, there exists an ultrafilter in $\mathcal{B}$ that contains $x$ and respects $\mathcal{T}$. 
This lemma was later proved by Tarski in a purely algebraic way. See [14] for a discussion of the role of the Baire category theorem in the proof.

\subsection{The Stone Representation Theorem}

Starting from a Boolean algebra $\mathcal{B}$, one can construct a topological space called a Stone space as follows. The points of the space are the ultrafilters of $\mathcal{B}$, which are in one-to-one correspondence with the Boolean algebra homomorphisms $\mathcal{B} \rightarrow \mathcal{L}$, where $\mathcal{2}$ is the two-element Boolean algebra. For each $x \in \mathcal{B}$, let $x^{\prime}$ be the set of ultrafilters containing $x$. The sets $x^{\prime}$ form a base for the topology. The resulting space is compact, Hausdorff, and totally disconnected. The basic open sets $x^{\prime}$ are both closed and open (clopen); spaces with a base of clopen sets are called zero-dimensional. For compact Hausdorff spaces, the notions of zero-dimensionality and total disconnectedness coincide.

A Stone space is defined to be a zero-dimensional compact Hausdorff space. The family of clopen sets of a Stone space form a Boolean algebra. One can go back and forth from Boolean algebras to Stone spaces; in both cases one obtains an object isomorphic to the starting object. Jonsson and Tarski [3] extended this to Boolean algebras with additional operators, essentially algebraic versions of modal operators, and corresponding topological spaces equipped with suitable closure operators.

The correspondence lifts to a contravariant equivalence between the category of Boolean algebras and Boolean algebra homomorphisms and the category of Stone spaces and continuous maps. Many other dualities in mathematics are recognized as being of this type [2].

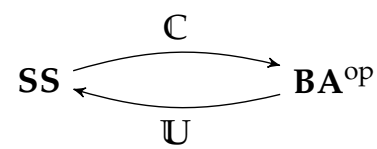

\section{Markov Processes and Markovian Logic}

\subsection{Markov Processes}

Markov processes (MPs) are models of probabilistic systems with a continuous state space and probabilistic transitions [12,20,23]. In earlier papers, 
they were called labeled Markov processes to emphasize the fact that there were multiple possible actions, but here we will suppress the labels, as they do not contribute any relevant structure for our results.

Definition 6 (Markov process). Given an analytic space $(M, \Sigma)$, a Markov process is a measurable mapping $\theta \in \llbracket M \rightarrow \Delta(M, \Sigma) \rrbracket$.

In what follows we identify a Markov process with the tuple $\mathcal{M}=$ $(M, \Sigma, \theta) ; M$ is the support set, denoted by $\operatorname{supp}(\mathcal{M})$, and $\theta$ is the transition function. For $m \in M, \theta(m): \Sigma \rightarrow[0,1]$ is a probability measure on the state space $(M, \Sigma)$. For $N \in \Sigma$, the value $\theta(m)(N) \in[0,1]$ represents the probability of a transition from $m$ to a state in $N$.

The condition that $\theta$ is a measurable function $\llbracket M \rightarrow \Delta(M, \Sigma) \rrbracket$ is equivalent to the condition that for fixed $N \in \Sigma$, the function $m \mapsto \theta(m)(N)$ is a measurable function $\llbracket M \rightarrow[0,1] \rrbracket$ (see e.g. Proposition 2.9 of [23]).

\subsection{Markovian Logic}

Markovian logic (ML) is a multi-modal logic for semantics based on MPs $[13,15,21,24-28]$. In addition to the Boolean operators, this logic is equipped with probabilistic modal operators $L_{r}$ for $r \in \mathbb{Q}_{0}$ that bound the probabilities of transitions. Intuitively, the formula $L_{r} \varphi$ is satisfied by $m \in \mathcal{M}$ whenever the probability of a transition from $m$ to a state satisfying $\varphi$ is at least $r$.

Definition 7 (Syntax). The formulas of $\mathcal{L}$ are defined, for a set $\mathcal{P}$ of atomic propositions, by the grammar

$$
\varphi::=p|\perp| \varphi \rightarrow \varphi \mid L_{r} \varphi
$$

where $p$ can be any element of $\mathcal{P}$ and $r$ any element of $\mathbb{Q}_{0}$.

The Boolean operators $\vee, \wedge, \neg$, and $\top$ are defined from $\rightarrow$ and $\perp$ as usual. For $r_{1}, \ldots, r_{n} \in \mathbb{Q}_{0}$ and $\varphi \in \mathcal{L}$, let

$$
L_{r_{1} \cdots r_{n}} \varphi=L_{r_{1}} \cdots L_{r_{n}} \varphi
$$

The Markovian semantics for $\mathcal{L}$ is defined for a given $\mathrm{MP} \mathcal{M}=(M, \Sigma, \theta)$ and $m \in M$, on top of an interpretation function $i: M \rightarrow 2^{\mathcal{P}}$, as follows.

- $\mathcal{M}, m, i \vDash p$ if $p \in i(m)$, 
- $\mathcal{M}, m, i \vDash \perp$ never,

- $\mathcal{M}, m, i \vDash \varphi \rightarrow \psi$ if $\mathcal{M}, m, i \vDash \psi$ whenever $\mathcal{M}, m, i \vDash \varphi$,

- $\mathcal{M}, m, i \vDash L_{r} \varphi$ if $\theta(m)(\llbracket \varphi \rrbracket) \geq r$, where $\llbracket \varphi \rrbracket=\{m \in M \mid \mathcal{M}, m, i \vDash \varphi\}$.

For the last clause to make sense, $\llbracket \varphi \rrbracket$ must be measurable. This is guaranteed by the fact that $\theta \in \llbracket M \rightarrow \Delta(M, \Sigma) \rrbracket$ (see e.g. [13]). It follows that

- $\mathcal{M}, m, i \vDash \top$ always,

- $\mathcal{M}, m, i \vDash \varphi \wedge \psi$ iff $\mathcal{M}, m, i \vDash \varphi$ and $\mathcal{M}, m, i \vDash \psi$,

- $\mathcal{M}, m, i \vDash \varphi \vee \psi$ iff $\mathcal{M}, m, i \vDash \varphi$ or $\mathcal{M}, m, i \vDash \psi$,

- $\mathcal{M}, m, i \vDash \neg \varphi$ iff not $\mathcal{M}, m, i \vDash \varphi$.

Given $\mathcal{M}=(M, \Sigma, \theta)$ and $i$, we say that $m \in M$ satisfies $\varphi$ if $\mathcal{M}, m, i \vDash \varphi$. We write $\mathcal{M}, m, i \not \models \varphi$ if it is not the case that $\mathcal{M}, m, i \vDash \varphi$; and we write $\mathcal{M}, m, i \vDash \Phi$ if $\mathcal{M}, m, i \vDash \varphi$ for all $\varphi \in \Phi$. We write $\Phi \vDash \varphi$ if $\mathcal{M}, m, i \vDash \varphi$ whenever $\mathcal{M}, m, i \vDash \Phi$. A formula or set of formulas is satisfiable if there exist an $\mathrm{MP} \mathcal{M}$, an interpretation function $i$ for $\mathcal{M}$ and $m \in \operatorname{supp}(\mathcal{M})$ that satisfies it. We say that $\varphi$ is valid and write $\vDash \varphi$ if $\varnothing \vDash \varphi$, that is, if $\neg \varphi$ is not satisfiable.

We now present an axiomatization of ML for Markovian semantics. The system is a Hilbert-style system consisting of the axioms and rules of propositional modal logic and the axioms and rules listed in Table 1. The axioms and the rules are stated for arbitrary $\varphi, \psi \in \mathcal{L}$ and arbitrary $r, s \in \mathbb{Q}_{0}$.

If $\Phi \subseteq \mathcal{L}$ and $\varphi \in \mathcal{L}$, we write $\Phi \vdash \varphi$ and say that $\Phi$ derives $\varphi$ if $\varphi$ is provable from the axioms and the extra assumptions $\Phi$. We write $\vdash \varphi$ if $\varnothing \vdash \varphi$.

A formula or set of formulas is consistent if it cannot derive $\perp$. We say that $\Phi \subseteq \mathcal{L}$ is maximally consistent if it is consistent and it has no proper consistent extensions.

The set $\Phi$ of formulas is filtered if for all $\varphi, \psi \in \Phi$ there exists $\rho \in \Phi$ with $\vdash \rho \rightarrow \varphi \wedge \psi$.

The (strong) completeness of this logic is proved in [15] by assuming Lindenbaum's lemma as a meta-axiom and using the following stronger 
(A1): $\vdash L_{0} \varphi$

(A2): $\vdash L_{r} T$

(A3): $\vdash L_{r} \varphi \rightarrow \neg L_{s} \neg \varphi, r+s>1$

(A4): $\vdash L_{r}(\varphi \wedge \psi) \wedge L_{s}(\varphi \wedge \neg \psi) \rightarrow L_{r+s} \varphi, r+s \leq 1$

(A5): $\vdash \neg L_{r}(\varphi \wedge \psi) \wedge \neg L_{s}(\varphi \wedge \neg \psi) \rightarrow \neg L_{r+s} \varphi, \quad r+s \leq 1$

(R1): $\frac{\vdash \varphi \rightarrow \psi}{\vdash L_{r} \varphi \rightarrow L_{r} \psi}$

(R2): $\left\{L_{r_{1} \cdots r_{n} r} \psi \mid r<s\right\} \vdash L_{r_{1} \cdots r_{n} s} \psi$

Table 1: The axioms of $\mathcal{L}$

version of (R1) for filtered $\Phi \subseteq \mathcal{L}$ :

$$
\frac{\Phi \vdash \varphi}{L_{r} \Phi \vdash L_{r} \varphi}
$$

where $L_{r} \Phi=\left\{L_{r} \psi \mid \psi \in \Phi\right\}$.

A consequence of our duality theorem is the (strong) completeness of ML with the axiomatization in Table 1.

\section{Aumann Algebras}

In this section we introduce an algebraic version of Markovian logic consisting of Boolean algebra with operators $F_{r}$ for $r \in \mathbb{Q}_{0}$ corresponding to the operators $L_{r}$ of ML. We call this Aumann Algebra (AA) in honor of Robert Aumann, who has made fundamental contributions to probabilistic logic $[24,25]$.

\subsection{Definition of Aumann Algebras}

Definition 8 (Aumann algebra). An Aumann algebra (AA) is a structure $\mathcal{A}=\left(A, \rightarrow, \perp,\left\{F_{r}\right\}_{r \in \mathbb{R}_{0}}, \leq\right)$ where 
- $(A, \rightarrow, \perp, \leq)$ is a Boolean algebra;

- for each $r \in \mathbb{Q}_{0}, F_{r}: A \rightarrow A$ is an unary operator; and

- the axioms in Table 2 hold for all $a, b \in A, r, s, r_{1}, \ldots, r_{n} \in \mathbb{Q}_{0}$.

The Boolean operations $\vee, \wedge, \neg$, and $\top$, are defined from $\rightarrow$ and $\perp$ as usual.

Morphisms of Aumann algebras are Boolean algebra homomorphisms that commute with the operations $F_{r}$. The category of Aumann algebras and Aumann algebra homomorphisms is denoted by AA.

We abbreviate $F_{r_{1}} \cdots F_{r_{n}} a$ by $F_{r_{1} \cdots r_{n}} a$.

(AA1) $\top \leq F_{0} a$

(AA2) $\top \leq F_{r} \top$

(AA3) $F_{r} a \leq \neg F_{s} \neg a, r+s>1$

(AA4) $F_{r}(a \wedge b) \wedge F_{s}(a \wedge \neg b) \leq F_{r+s} a, r+s \leq 1$

(AA5) $\neg F_{r}(a \wedge b) \wedge \neg F_{s}(a \wedge \neg b) \leq \neg F_{r+s} a, r+s \leq 1$

(AA6) $a \leq b \Rightarrow F_{r} a \leq F_{r} b$

(AA7) $\left(\bigwedge_{r<s} F_{r_{1} \cdots r_{n} r} a\right)=F_{r_{1} \cdots r_{n} s} a$

Table 2: Aumann algebra

The operator $F_{r}$ is the algebraic counterpart of the logical modality $L_{r}$. The first two axioms state tautologies, while the third captures the way $F_{r}$ interacts with negation. Axioms (AA4) and (AA5) assert finite additivity, while (AA6) asserts monotonicity.

The most interesting axiom is the infinitary axiom (AA7). It asserts that $F_{r_{1} \cdots r_{n} s} a$ is the greatest lower bound of the set $\left\{F_{r_{1} \cdots r_{n} r} a \mid r<s\right\}$ with respect to the natural order $\leq$. In SMPs, it will imply countable additivity.

The following lemma establishes some basic consequences.

Lemma 9. Let $\mathcal{A}=\left(A, \rightarrow, \perp,\left\{F_{r}\right\}_{r \in \mathbb{Q}_{0}}, \leq\right)$ be an Aumann algebra. For all $a, b \in A$ and $r, s \in \mathbb{Q}_{0}$,

(i) $F_{r} \perp=\perp$ for $r>0$; 
(ii) if $r \leq s$, then $F_{s} a \leq F_{r} a$;

(iii) if $a \leq \neg b$ and $r+s>1$, then $F_{r} a \leq \neg F_{s} b$.

As expected, the formulas of Markovian logic modulo logical equivalence form a free countable Aumann algebra. Define $\equiv$ on formulas by: $\varphi \equiv \psi$ if $\vdash \varphi \rightarrow \psi$ and $\vdash \psi \rightarrow \varphi$. Let $[\varphi]$ denote the equivalence class of $\varphi$ modulo $\equiv$, and let $\mathcal{L} / \equiv=\{[\varphi] \mid \varphi \in \mathcal{L}\}$. By (R1), the modality $L_{r}$ is well defined on $\equiv$-classes. The Boolean operators are also well defined by considerations of propositional logic.

Theorem 10. The structure

$$
\left(\mathcal{L} / \equiv, \rightarrow,[\perp],\left\{L_{r}\right\}_{r \in \mathbb{Q}_{0}}, \leq\right)
$$

is an Aumann algebra, where $[\varphi] \leq[\psi]$ iff $\vdash \varphi \rightarrow \psi$.

Proof. The axioms of Boolean algebras are trivially satisfied.

Now we have to verify the axioms in Table 2 for $F_{r}=L_{r}$.

(AA1) in this setting corresponds to $\vdash T \rightarrow L_{0} \varphi$, which is equivalent to $\vdash L_{0} \varphi$ and an instance of axiom (A1) of $\mathcal{L}$.

Similarly, (AA2), (AA3), (AA4) and (AA5) are easily seen to correspond to axioms (A2), (A3), (A4) and (A5) respectively. (AA6) and (AA7) are instances of the rules (R1) and (R2) respectively. Consequently all the axioms of Aumann algebra are verified.

Moreover, $\mathcal{L}$ is countable.

\subsection{Extended Satisfiability Principles for Aumann Algebras}

We saw in the previous section that the formulas of ML give rise to an Aumann algebra. We now define a satisfiability relation for AAs that generalizes the satisfiability relation for ML and prove the corresponding soundness result for MPs.

Let $\mathcal{M}=(M, \Sigma, \theta)$ be a MP and let $\llbracket \cdot \rrbracket$ be an interpretation of terms in the language of Aumann algebras as measurable sets in $M$ in which the Boolean operators have their usual set-theoretic interpretation and for $S \in$ $\Sigma$ and $t \in \mathbb{Q}_{0}$,

$$
\llbracket F_{t} \rrbracket(S)=\{m \in M \mid \theta(m)(S) \geq t\} \quad \llbracket F_{t} a \rrbracket=\llbracket F_{t} \rrbracket(\llbracket a \rrbracket) .
$$


If $\mathcal{A}$ is freely generated, then an interpretation function is defined by any function that associates to each generator of $\mathcal{A}$ a subset of $M$.

Lemma 11. $\llbracket F_{t} \rrbracket$ preserves meets of countable measurable chains; that is, if $S_{i}$, $i \in I$ is a countable chain of measurable sets, then

$$
\llbracket F_{t} \rrbracket\left(\bigcap_{i \in I} S_{i}\right)=\bigcap_{i \in I} \llbracket F_{t} \rrbracket\left(S_{i}\right) .
$$

Proof. We have

$$
\begin{aligned}
\llbracket F_{t} \rrbracket\left(\bigcap_{i \in I} S_{i}\right) & =\left\{m \in M \mid \theta(m)\left(\bigcap_{i \in I} S_{i}\right) \geq t\right\} \\
& =\left\{m \in M \mid \inf _{i \in I} \theta(m)\left(S_{i}\right) \geq t\right\} \\
& =\left\{m \in M \mid \forall i \in I, \theta(m)\left(S_{i}\right) \geq t\right\} \\
& =\bigcap_{i \in I}\left\{m \in M \mid \theta(m)\left(S_{i}\right) \geq t\right\} \\
& =\bigcap_{i \in I} \llbracket F_{t} \rrbracket\left(S_{i}\right) .
\end{aligned}
$$

We can prove now that the axioms of Aumann algebras are sound for Markov processes.

Theorem 12 (Soundness). Let $\mathcal{A}$ be an Aumann algebra and $a \in \mathcal{A}$. If $T \leq a$, then for any Markov process $\mathcal{M}=(M, \Sigma, \theta)$ and any interpretation $\llbracket \cdot \rrbracket$ of terms in the language of Aumann algebras as measurable sets in $M$ with the properties listed above, $\llbracket a \rrbracket=M$.

Proof. As in the case of soundness for logics, we prove the soundness by showing that each axiom is satisfied by any Markov process. In the following, we only prove it for (AA7).

Let $\mathcal{F}$ be the set of terms of the form $\alpha^{r}=F_{t_{1} \cdots t_{n} r} a$ for $a \in A$ and $0 \leq$ $t_{1}, \ldots, t_{n}, r \leq 1$. We consider this formula parameterized by $r$; that is, if $\alpha^{r}=F_{t_{1} \cdots t_{n} r} a$, then $\alpha^{s}$ denotes $F_{t_{1} \cdots t_{n} s} a$. The axiomatization of AAs includes all infinitary conditions of the form

$$
\alpha^{s}=\bigwedge_{r<s} \alpha^{r} .
$$


To prove the soundness of (AA7), it is sufficient to prove that

$$
\llbracket \alpha^{s} \rrbracket=\llbracket \bigwedge_{r<s} \alpha^{r} \rrbracket .
$$

We proceed by induction on $n$. For the basis,

$$
\begin{aligned}
\llbracket F_{s} a \rrbracket & =\llbracket F_{s} \rrbracket(\llbracket a \rrbracket)=\{u \mid \theta(u)(\llbracket a \rrbracket) \geq s\} \\
& =\{u \mid \forall r<s \theta(u)(\llbracket a \rrbracket) \geq r\}=\bigcap_{r<s}\{u \mid \theta(u)(\llbracket a \rrbracket) \geq r\} \\
& =\bigcap_{r<s} \llbracket F_{r} a \rrbracket=\llbracket \bigwedge_{r<s} F_{r} a \rrbracket .
\end{aligned}
$$

For the induction step, if $\llbracket \alpha^{s} \rrbracket=\llbracket \wedge_{r<s} \alpha^{r} \rrbracket$ and $t>0$,

$$
\begin{aligned}
\llbracket F_{t} \alpha^{s} \rrbracket & =\llbracket F_{t} \rrbracket\left(\llbracket \alpha^{s} \rrbracket\right)=\llbracket F_{t} \rrbracket\left(\llbracket \bigwedge_{r<s} \alpha^{r} \rrbracket\right) \\
& =\llbracket F_{t} \rrbracket\left(\bigcap_{r<s} \llbracket \alpha^{r} \rrbracket\right)=\bigcap_{r<s} \llbracket F_{t} \rrbracket\left(\llbracket \alpha^{r} \rrbracket\right) \text { by Lemma } 11 \\
& =\bigcap_{r<s} \llbracket F_{t} \alpha^{r} \rrbracket=\llbracket \bigwedge_{r<s} F_{t} \alpha^{r} \rrbracket .
\end{aligned}
$$

\section{Stone Markov Processes}

In our duality theory, we work with Markov processes constructed from certain zero-dimensional Hausdorff spaces. We call such structures StoneMarkov processes (SMPs).

\subsection{MPs with Distinguished Base}

We restrict our attention to Markov processes $(M, \mathcal{A}, \theta)$, where $\mathcal{A}$ is a distinguished countable base of clopen sets that is closed under the set-theoretic Boolean operations and the operations

$$
F_{r}(A)=\{m \mid \theta(m)(A) \geq r\}, \quad r \in \mathbb{Q}_{0} .
$$

The measurable sets $\Sigma$ are the Borel sets of the topology generated by $\mathcal{A}$. Morphisms of such spaces are required to preserve the distinguished base; thus a morphism $f: \mathcal{M} \rightarrow \mathcal{N}$ is a continuous function such that 
- for all $m \in M$ and $B \in \Sigma_{\mathcal{N}}$,

$$
\theta_{\mathcal{M}}(m)\left(f^{-1}(B)\right)=\theta_{\mathcal{N}}(f(m))(B) ;
$$

- for all $A \in \mathcal{A}_{\mathcal{N}}, f^{-1}(A) \in \mathcal{A}_{\mathcal{M}}$.

\subsection{Saturation}

Unlike Stone spaces, SMPs are not topologically compact, but we do postulate a completeness property that is a weak form of compactness, which we call saturation. One can saturate a given SMP by a completion procedure that is reminiscent of Stone-Čech compactification. Intuitively, one adds points to the structure without changing the represented algebra. An $\mathrm{MP}$ is saturated if it is maximal with respect to this operation. The term comes from a related concept of the same name in model theory [29, Ch. 5].

Formally, consider MP morphisms $f: \mathcal{M} \rightarrow \mathcal{N}$ such that

- $f$ is a homeomorphism between $\mathcal{M}$ and its image in $\mathcal{N}$;

- the image $f(M)$ is dense in $N$; and

- $f$ preserves the distinguished base in the forward direction as well as the backward; that is, if $A \in \mathcal{A}_{\mathcal{M}}$, then there exists $B \in \mathcal{A}_{\mathcal{N}}$ such that $A=f^{-1}(B)$.

Call such a morphism a strict embedding. The collection of all $\mathcal{N}$ such that there exists a strict embedding $\mathcal{M} \rightarrow \mathcal{N}$ contains a final object, which is the colimit of the strict embeddings $\mathcal{M} \rightarrow \mathcal{N}$. This is the saturation of $\mathcal{M}$.

One can construct the saturation by completing by a certain family of ultrafilters called good ultrafilters. These are ultrafilters respecting the infinitary condition (AA7) in the definition of Aumann algebras (§4). All ultrafilters represented by a point of an SMP are already good, and one must only add the rest. The details of this construction are given in $\S 6$.

\subsection{Definition of SMP}

Definition 13 (Stone-Markov Process). A Markov process $\mathcal{M}=(M, \mathcal{A}, \theta)$ with distinguished base is a Stone-Markov process (SMP) if it is saturated. 
The morphisms of SMPs are just the morphisms of MPs with distinguished base as defined above.

The category of SMPS and SMP morphisms is denoted SMP.

\section{Stone Duality}

In this section we describe the duality between SMPs and countable AAs. This is in the spirit of the classical Stone representation theorem [1], or, more precisely, the representation theorem of Jonsson and Tarski [3] for Boolean algebras with operators. Here the details are somewhat different, as we must deal with measure theory.

\subsection{From AAs to SMPs}

For this subsection, we fix an arbitrary countable Aumann algebra

$$
\mathcal{A}=\left(A, \rightarrow, \perp,\left\{F_{r}\right\}_{r \in \mathbb{Q}_{0}}, \leq\right) .
$$

Let $\mathcal{U}^{*}$ be the set of all ultrafilters of $\mathcal{A}$. The classical Stone construction gives a Boolean algebra of sets isomorphic to $\mathcal{A}$ with elements

$$
(a)^{*}=\left\{u \in \mathcal{U}^{*} \mid a \in u\right\}, a \in \mathcal{A} \quad(\mid \mathcal{A})^{*}=\left\{(a)^{*} \mid a \in A\right\} .
$$

The sets $(a)^{*}$ generate a Stone topology $\tau^{*}$ on $\mathcal{U}^{*}$, and the sets $(a)^{*}$ are exactly the clopen sets of the topology.

Let $\mathcal{F}$ be the set of elements of the form $\alpha^{r}=F_{t_{1} \cdots t_{n} r} a$ for $a \in A$ and $t_{1}, \ldots, t_{n}, r \in \mathbb{Q}_{0}$. As before, we consider this term as parameterized by $r$; that is, if $\alpha^{r}=F_{t_{1} \cdots t_{n} r} a$, then $\alpha^{s}$ denotes $F_{t_{1} \cdots t_{n} s} a$. The set $\mathcal{F}$ is countable since $A$ is. Axiom (AA7) asserts all infinitary conditions of the form

$$
\alpha^{s}=\bigwedge_{r<s} \alpha^{r}
$$

for $\alpha^{s} \in \mathcal{F}$. Let us call an ultrafilter $u$ bad if it violates one of these conditions in the sense that for some $\alpha^{s} \in \mathcal{F}, \alpha^{r} \in u$ for all $r<s$ but $\alpha^{s} \notin u$. Otherwise, $u$ is called good. Let $\mathcal{U}$ be the set of good ultrafilters of $\mathcal{A}$.

Let $\tau=\left\{B \cap \mathcal{U} \mid B \in \tau^{*}\right\}$ be the subspace topology on $\mathcal{U}$, and let

$$
(\mid a)=\{u \in \mathcal{U} \mid a \in \mathcal{U}\}=(\mid a)^{*} \cap \mathcal{U} \quad(\mathcal{A})=\{(\mid a) \mid a \in A\} .
$$


Then $\tau$ is countably generated by the sets $(a)$ and all $(a)$ are clopen in the subspace topology.

The next lemma asserts that the set $\mathcal{U} \backslash \mathcal{U}^{*}$ of bad ultrafilters is meager. We will use this to argue that $\mathcal{U}$ is dense in $\mathcal{U}^{*}$, therefore no $(a)$ vanishes as a result of dropping the bad points from $(a)^{*}$. It will follow that $(\mathcal{A})$ and $(\mathcal{A})^{*}$ are isomorphic as Boolean algebras.

Lemma 14. The set $\mathcal{U}^{*} \backslash \mathcal{U}$ is of first category in the Stone topology $\tau^{*}$.

Proof. We must prove that $\mathcal{U}^{*} \backslash \mathcal{U}$ is a countable union of nowhere dense sets. Since $A$ countable, the set $\mathcal{F}$ is countable as well. Each bad ultrafilter $u \in \mathcal{U}^{*} \backslash \mathcal{U}$ violates at least one constraint (1), thus

$$
\mathcal{U}^{*} \backslash \mathcal{U}=\bigcup_{\alpha^{s} \in \mathcal{F}} U_{\alpha^{s}}
$$

where

$$
\begin{aligned}
U_{\alpha^{s}} & =\left\{u \in \mathcal{U}^{*} \mid \alpha^{s} \notin u \text { and } \forall r<s \alpha^{r} \in u\right\} \\
& =\left(\neg \alpha^{s}\right)^{*} \backslash \bigcup_{r<s}\left(\neg \alpha^{r} D^{*} .\right.
\end{aligned}
$$

Now we argue that each $U_{\alpha^{s}}$ is nowhere dense. In $\tau^{*},\left(\neg \alpha^{s}\right)^{*}$ is a closed set while $\bigcup_{r<s}\left(\neg \alpha^{r}\right)^{*}$ is a countable union of open sets, hence open. Moreover,

$$
\left(\neg \alpha^{s}\right)^{*}=\overline{\bigcup_{r<s}\left(\neg \alpha^{r}\right)^{*}},
$$

where $\bar{S}$ denotes the closure of $S$. Consequently, $U_{\alpha^{s}}$ is the boundary of an open set, hence nowhere dense.

In the remainder of this section, we show that the topological space $(\mathcal{U}, \tau)$ is a zero-dimensional Hausdorff space with base $(\mathcal{A})$ and that this space gives rise to an $\operatorname{SMP}(\mathcal{U},(\mathcal{A}), \theta)$. Note that unlike $\left(\mathcal{U}^{*}, \tau^{*}\right)$, the space $(\mathcal{U}, \tau)$ is not compact, but it is saturated.

In order to prove these claims, we need to focus on the properties of the subspace topology. Recall that for a topological space $(X, \tau)$ and an arbitrary set $Y \subseteq X$, we can view $Y$ as a topological space with the relative topology $\tau^{\prime}=\{u \cap Y \mid u \in \tau\}$. Moreover, if $u$ is an open (closed) set in $\tau$, then $u \cap Y$ is open (closed) in $\tau^{\prime}$, and if $B \subseteq \tau$ is a base for $\tau$, then $B^{\prime}=\{u \cap Y \mid u \in B\}$ is a base for $\tau^{\prime}$.

The next lemma is immediate from the definitions. 
Lemma 15. Any subspace of a zero-dimensional space is zero-dimensional, and any subspace of a Hausdorff space is Hausdorff.

Proof. Let $(X, \tau)$ space and $\left(Y, \tau^{\prime}\right)$ a subspace with the relative topology. Suppose that $(X, \tau)$ is zero-dimensional. For all $u^{\prime} \in \tau^{\prime}$, there exists $u \in \tau$ such that $u^{\prime}=u \cap Y$. Since $(X, \tau)$ has a base of clopen sets, we have $u=$ $\bigcup_{i \in I} u_{i}$, where the $u_{i}$ are an indexed collection of clopens in $\tau$. Then

$$
u^{\prime}=\left(\bigcup_{i \in I} u_{i}\right) \cap Y=\bigcup_{i \in I}\left(u_{i} \cap Y\right)
$$

Since the $u_{i}$ are clopen in $\tau$, the $u_{i} \cap Y$ are clopen in $\tau^{\prime}$, thus the clopens of $\tau^{\prime}$ form a base for the topology $\tau^{\prime}$, therefore $\left(Y, \tau^{\prime}\right)$ is zero-dimensional.

If $(X, \tau)$ is Hausdorff, then for all $x, y \in Y$ there exist disjoint $u, v \in \tau$ such that $x \in u$ and $y \in v$. Then $x \in u \cap Y, y \in v \cap Y$, and $u \cap Y$ and $v \cap Y$ are disjoint and in $\tau^{\prime}$. As $x, y$ were arbitrary, $\left(Y, \tau^{\prime}\right)$ is Hausdorff.

Since $(\mathcal{U}, \tau)$ is a subspace of the Stone space $\left(\mathcal{U}^{*}, \tau^{*}\right)$, the next proposition is just an instance of Lemma 15.

Proposition 16. The space $(\mathcal{U}, \tau)$ is a zero-dimensional Hausdorff space.

\subsection{Construction of $\mathbb{M}(\mathcal{A})$}

We can now form a Markov process $\mathbb{M}(\mathcal{A})=(\mathcal{U}, \Sigma, \theta)$, where $\Sigma$ is the $\sigma$-algebra generated by $(\mathcal{A})$. To define the measure $\theta(u)$ for an ultrafilter $u \in \mathcal{U}$, we need to prove some additional results.

Lemma 17. For all $a \in A$ and $u \in \mathcal{U}$, the set

$$
\left\{r \in \mathbb{Q}_{0} \mid F_{r} a \in u\right\}
$$

is nonempty and closed downward in the natural order on $\mathbb{Q}_{0}$.

Proof. The set contains at least 0 by (AA1). Downward closure follows from Lemma 9(ii). 
It follows that $\left\{r \in \mathbb{Q}_{0} \mid \neg F_{r} a \in u\right\}$ is closed upward. Thus we can define the function $\theta: \mathcal{U} \rightarrow(\mathcal{A}) \rightarrow[0,1]$ by

$$
\begin{aligned}
\theta(u)((\mid a)) & =\sup \left\{r \in \mathbb{Q}_{0} \mid F_{r} a \in u\right\} \\
& =\inf \left\{r \in \mathbb{Q}_{0} \mid \neg F_{r} a \in u\right\} .
\end{aligned}
$$

Note that $\theta(u)((a))$ is not necessarily rational. In the following, we use Theorem 3 to show that $\theta$ can be uniquely extended to a transition function. This will allow us to construct a Markov process on the space of good ultrafilters.

Lemma 18. The set $(\mid \mathcal{A})$ is a field of sets, and for all $u \in \mathcal{U}$, the function $\theta(u)$ is finitely additive.

Proof. That the set $(\mathcal{A})$ is a field of sets is immediate from the Stone representation theorem and the fact that $(\mathcal{A})$ is dense in $(\mathcal{A})^{*}$.

To show finite additivity, suppose $a, b \in A$ and $(a) \cap(b)=\varnothing$. Then $a \wedge b=0$. We wish to show that

$$
\theta(u)((\mid a \vee b))=\theta(u)((\mid a))+\theta(u)((\mid b)) .
$$

It suffices to show the inequality in both directions. For $\leq$, by the definition of $\theta$, it suffices to show

$$
\begin{aligned}
& \sup \left\{t \mid F_{t}(a \vee b) \in u\right\} \\
& \leq \inf \left\{r \mid \neg F_{r} a \in u\right\}+\inf \left\{s \mid \neg F_{s} b \in u\right\} \\
& =\inf \left\{r+s \mid \neg F_{r} a \in u \text { and } \neg F_{s} b \in u\right\} \\
& =\inf \left\{r+s \mid \neg F_{r} a \wedge \neg F_{s} b \in u\right\} ;
\end{aligned}
$$

that is, if $F_{t}(a \vee b) \in u$ and $\neg F_{r} a \wedge \neg F_{s} b \in u$, then $t \leq r+s$. But

$$
\begin{aligned}
\neg F_{r} a \wedge \neg F_{s} b & =\neg F_{r}((a \vee b) \wedge a) \wedge \neg F_{s}((a \vee b) \wedge \neg a) \\
& \leq \neg F_{r+s}(a \vee b) \quad \text { by }(\mathrm{AA} 5),
\end{aligned}
$$

thus $\neg F_{r+s}(a \vee b) \in u$, and $t \leq r+s$ follows from the characterization of Lemma 17.

The inequality in the opposite direction is similar, using (AA4). We need to show

$$
\inf \left\{t \mid \neg F_{t}(a \vee b) \in u\right\} \geq \sup \left\{r+s \mid F_{r} a \wedge F_{s} b \in u\right\}
$$


that is, if $\neg F_{t}(a \vee b) \in u$ and $F_{r} a \wedge F_{s} b \in u$, then $t \geq r+s$. But

$$
\begin{aligned}
F_{r} a \wedge F_{s} b & =F_{r}((a \vee b) \wedge a) \wedge F_{s}((a \vee b) \wedge \neg a) \\
& \leq F_{r+s}(a \vee b) \quad \text { by }(\mathrm{AA} 4),
\end{aligned}
$$

thus $F_{r+s}(a \vee b) \in u$, and again $r+s \leq t$ by Lemma 17 .

The following is the key technical lemma where we use the fact that we have removed the bad ultrafilters.

Lemma 19. For $u \in \mathcal{U}, \theta(u)$ is continuous from above at $\varnothing$.

Proof. We prove that if $u \in \mathcal{U}$ (it is a good ultrafilter) and $b_{0} \geq b_{1} \geq \cdots$ with $\bigcap_{i}\left(b_{i}\right)=\varnothing$, then

$$
\inf _{i} \theta(u)\left(\left(b_{i}\right)\right)=0 .
$$

Consider the countable set $\mathcal{F}$ of elements of the form $\alpha^{r}=F_{t_{1} \cdots t_{n} r} a$ for $a \in A$ and rational $t_{1}, \ldots, t_{n}, r \geq 0$, parameterized by $r$. If $r<s$, then $\alpha^{\mathcal{S}} \leq \alpha^{r}$. Using (AA4),

$$
\theta(u)\left(\left(\alpha^{r} \wedge \neg \alpha^{s}\right\rangle\right) \leq \theta(u)\left(\left(\alpha^{r}\right\rangle\right)-\theta(u)\left(\left(\left|\alpha^{s}\right\rangle\right) .\right.
$$

Since $u$ is good, $F_{t} \alpha^{r} \in u$ for all $r<s$ iff $F_{t} \alpha^{s} \in u$, therefore

$$
\theta(u)\left(\left(\mid \alpha^{s}\right)\right)=\inf _{r<s} \theta(u)\left(\left(\left|\alpha^{r}\right\rangle\right) .\right.
$$

Let $\varepsilon>0$ be an arbitrarily small positive number. For each $\alpha \in \mathcal{F}$ and $s \in \mathbb{Q}_{0}$, choose $\varepsilon_{\alpha}^{s}>0$ such that $\sum_{\alpha \in \mathcal{F}} \sum_{s \in \mathbb{Q}_{0}} \varepsilon_{\alpha}^{s}=\varepsilon$. By (2) and (3), we can choose $r_{\alpha}^{s}<s$ such that

$$
\theta(u)\left(\left(\alpha^{r_{\alpha}^{s}} \wedge \neg \alpha^{s}\right\rangle\right) \leq \theta(u)\left(\left(\alpha^{r_{\alpha}^{s}}\right)\right)-\theta(u)\left(\left(\left|\alpha^{s}\right\rangle\right) \leq \varepsilon_{\alpha}^{s} .\right.
$$

The assumption $\bigcap_{i}\left(\mid b_{i}\right)=\varnothing$ implies that $\bigcap_{i} \mid\left(b_{i}\right)^{*}$ contains only bad ultrafilters. The set of good ultrafilters is

$$
\bigcap_{\alpha \in \mathcal{F}} \bigcap_{s \in \mathbb{Q}_{0}}\left(\bigcup_{r<s}\left(\neg \neg \alpha^{r}\right)^{*} \cup\left(\alpha^{s}\right)^{*}\right) .
$$

Thus $\bigcap_{i}\left(\mid b_{i}\right)=\varnothing$ is equivalent to the condition

$$
\left(\bigcap_{\alpha \in \mathcal{F}} \bigcap_{s \in \mathbb{Q}_{0}}\left(\bigcup_{r<s}\left(\neg \neg \alpha^{r}\right)^{*} \cup\left(\mid \alpha^{s}\right)^{*}\right)\right) \cap \bigcap_{i}\left(b b_{i}\right)^{*}=\varnothing .
$$


From this it follows that

$$
\left.\left(\bigcap_{\alpha \in \mathcal{F}} \bigcap_{s \in \mathbb{Q}_{0}}\left(\mid \neg \alpha^{r_{\alpha}^{s}}\right)^{*} \cup\left(\alpha^{s}\right)^{*}\right)\right) \cap \bigcap_{i}\left(b b_{i}\right)^{*}=\varnothing .
$$

Since the space of ultrafilters is compact in the presence of the bad ultrafilters and $(a)^{*}$ is a clopen for any $a \in A$, there exist finite sets $C_{0} \subseteq \mathcal{F}$ and $S_{0} \subseteq \mathbb{Q} \cap[0,1]$ and $j \in \mathbb{N}$ such that

$$
\bigcap_{\alpha \in C_{0}} \bigcap_{s \in S_{0}}\left(\neg \alpha^{r_{\alpha}^{s}} \vee \alpha^{s}\right)^{*} \cap\left(b_{j}\right)^{*}=\varnothing,
$$

or in other words,

$$
\begin{aligned}
\left(b_{j}\right)^{*} & \subseteq \bigcup_{\alpha \in C_{0}} \bigcup_{s \in S_{0}}\left(\alpha^{r_{\alpha}^{s}} \wedge \neg \alpha^{s}\right)^{*} \\
& =0 \bigvee_{\alpha \in C_{0}} \bigvee_{s \in S_{0}}\left(\alpha^{r_{\alpha}^{s}} \wedge \neg \alpha^{s}\right) D^{*}
\end{aligned}
$$

Thus in the Boolean algebra $\mathcal{A}$,

$$
b_{j} \leq \bigvee_{\alpha \in C_{0}} \bigvee_{s \in S_{0}}\left(\alpha^{r_{\alpha}^{s}} \wedge \neg \alpha^{s}\right)
$$

Consequently,

$$
\begin{aligned}
\theta(u)\left(\left(\mid b_{j}\right)\right) & \left.\leq \theta(u)\left(0 \bigvee_{\alpha \in C_{0}} \bigvee_{s \in S_{0}}\left(\alpha^{r_{\alpha}^{s}} \wedge \neg \alpha^{s}\right)\right)\right) \\
& \leq \sum_{\alpha \in C_{0}} \sum_{s \in S_{0}} \theta(u)\left(\left(\alpha^{r_{\alpha}^{s}} \wedge \neg \alpha^{s}\right)\right) \\
& \leq \sum_{\alpha \in C_{0}} \sum_{s \in S_{0}} \varepsilon_{\alpha}^{s} \leq \varepsilon .
\end{aligned}
$$

As $\varepsilon>0$ was arbitrary, $\left.\inf _{i} \theta(u)\left(0 b_{i}\right)\right)=0$.

Since $(\mathcal{A})$ is a field, the previous results and Theorem 3 imply that for all $u \in \mathcal{U}$, the set function $\theta(u)$ can be uniquely extended to a measure on the $\sigma$-algebra $\Sigma$ generated by $(\mathcal{A})$.

Now we are ready to prove that $\mathbb{M}(\mathcal{A})$ is a Stone Markov process.

Theorem 20. If $\mathcal{A}$ is a countable Aumann algebra, then $\mathbb{M}(\mathcal{A})=(\mathcal{U},(\mathcal{A}), \theta)$ is a Stone Markov process. 
Proof. We first prove that the space of good ultrafilters is analytic. Since any Stone space is Polish, the set of all ultrafilters (good and bad) is Polish. The good ultrafilters form a Borel set in the space of all ultrafilters-in fact, a $G_{\sigma \delta}$ Borel set as given by (4)—and since any Borel set in a Polish space is analytic, we obtain that the space of good ultrafilters is analytic.

The space is saturated, since all possible good ultrafilters are present, and the set $\{(a) \mid u \in(a)\}$ is just $u$.

To conclude that $\mathbb{M}(\mathcal{A})$ is a Markov process, it remains to verify that $\theta$ is a measurable function. Let $a \in A, r \in \mathbb{R} \cap[0,1]$, and $\left(r_{i}\right)_{i} \subseteq \mathbb{Q}_{0}$ an increasing sequence with supremum $r$. Let $X=\{\mu \in \Delta(\mathcal{U}, \Sigma) \mid \mu((\mid a)) \geq$ $r\}$. It suffices to prove that $\theta^{-1}(X) \in \Sigma$. But

$$
\begin{aligned}
\theta^{-1}(X) & =\{u \in \mathcal{U} \mid \theta(u)((|a|)) \geq r\} \\
& =\bigcap_{i}\left\{u \in \mathcal{U} \mid \theta(u)\left((|a|) \geq r_{i}\right\}\right. \\
& =\bigcap_{i}\left(F_{r_{i}} a\right) \in \Sigma .
\end{aligned}
$$

Now we are ready to prove the algebraic version of a truth lemma for Aumann algebras.

Lemma 21 (Extended Truth Lemma). Let $\mathcal{A}$ be a countable Aumann algebra and $\llbracket \cdot \rrbracket$ an interpretation of elements of $\mathcal{A}$ as measurable sets in $\mathcal{M}$ such that for any generator $p$ of $\mathcal{A}, \llbracket p \rrbracket=\{u \in \mathcal{U} \mid p \in u\}$. Then, for arbitrary $a \in A$,

$$
\llbracket a \rrbracket=(a) .
$$

Proof. We prove it by induction on the structure of the element $a$.

The case $a=p$, where $p$ is a generator of $\mathcal{A}$ :

$$
\llbracket a \rrbracket=\{u \in \mathcal{U} \mid u \ni p\}=(p) .
$$

The Boolean cases are trivial.

The case $a=F_{r} b$ :

$$
\begin{gathered}
\llbracket F_{r} b \rrbracket=\llbracket F_{r} \rrbracket(\llbracket b \rrbracket)=\{u \in \mathcal{U} \mid \theta(u)(\llbracket b \rrbracket) \geq r\}= \\
\{u \in \mathcal{U} \mid \theta(u)((b b)) \geq r\}=\left(\mid F_{r} b\right) .
\end{gathered}
$$




\subsection{From SMPs to AAs}

Let $\mathcal{M}=(M, \mathcal{B}, \theta)$ be a Stone Markov process with distinguished base $\mathcal{B}$. By definition, $\mathcal{B}$ is a field of clopen sets closed under the operations

$$
F_{r}(A)=\{m \in M \mid \theta(m)(A) \geq r\} .
$$

Theorem 22. The structure $\mathcal{B}$ with the set-theoretic Boolean operations and the operations $F_{r}, r \in \mathbb{Q}_{0}$, is a countable Aumann algebra.

We denote this algebra by $\mathbb{A}(\mathcal{M})$.

Proof. We verify all the axioms of Aumann algebra.

(AA1) has the form $M \subseteq F_{0} c$ for arbitrary $c \in \mathcal{B}$. Trivially true, since $F_{0} c=\{m \in M \mid \theta(m)(c) \geq 0\}=M$.

(AA2) has the form $M \subseteq F_{r} M$ that is trivially true, since $\theta(m)(M)=1$ implies $F_{r} M=\{m \in M \mid \theta(m)(M) \geq r\}=M$.

(AA3) has the form $F_{r} c \subseteq M \backslash F_{s}(M \backslash c)$ for arbitrary $c \in \mathcal{B}$ and $r+s>$ 1. This is equivalent to $F_{r} c \cap F_{s}(M \backslash c)=\varnothing$ for $s+t>1$.

Note that $F_{s}(M \backslash c)=\{m \in M \mid \theta(m)(M \backslash c) \geq s\}=\{m \in M \mid \theta(m)(c) \leq$ $1-s\}$. If there exists $m \in F_{r} c \cap F_{s}(M \backslash c)$, then using the previous observation we should have $r \leq \theta(m)(c) \leq 1-s$, implying $r \leq 1-s$, but this contradicts the fact that $r+s>1$.

(AA4) has the form $F_{r}\left(c \cap c^{\prime}\right) \cap F_{s}\left(c \cap\left(M \backslash c^{\prime}\right)\right) \subseteq F_{r+s} c$. Observe that $F_{r}\left(c \cap c^{\prime}\right) \cap F_{s}\left(c \cap\left(M \backslash c^{\prime}\right)\right)=\left\{m \in M \mid \theta(m)\left(c \cap c^{\prime}\right) \geq r\right.$ and $\theta(m)(c \cap(M \backslash$ $\left.\left.\left.c^{\prime}\right)\right) \geq s\right\}$. Using additivity we obtain further that $F_{r}\left(c \cap c^{\prime}\right) \cap F_{s}(c \cap(M \backslash$ $\left.\left.c^{\prime}\right)\right)=\{m \in M \mid \theta(m)(c) \geq r+s\}=F_{r+s} c$.

(AA5) has the form $\left(M \backslash F_{r}\left(c \cap c^{\prime}\right)\right) \cap\left(M \backslash F_{s}\left(c \cap\left(M \backslash c^{\prime}\right)\right)\right) \subseteq M \backslash F_{r+s} c$ and can be proved as the case of (AA4).

(AA6) has the form $c \subseteq c^{\prime}$ implies $F_{r} c \subseteq F_{r} c^{\prime}$. This follows from the monotonicity of $\theta(m)$.

(AA7) has the form $\bigcap_{r<s} F_{r_{1}, \ldots, r_{n}, r} c=F_{r_{1}, \ldots, r_{n}, s} \mathcal{c}$. The proof is done inductively on $n$.

For $n=0$ we need to prove that $\bigcap_{r<s} F_{r} c=F_{s} c$. Observe that $\bigcap_{r<s} F_{r} c=\{m \in$ $M \mid \theta(m)(c) \leq r$ for all $r>s\}=\{m \in M \mid \theta(m)(c) \leq s\}=F_{s} c$.

The inductive step uses (AA6). 


\subsection{Duality}

In this section we summarize the previous results in the form of the duality theorem.

Theorem 23 (Duality Theorem).

(i) Any countable Aumann algebra $\mathcal{A}$ is isomorphic to $\mathbb{A}(\mathbb{M}(\mathcal{A}))$ via the map $\beta: \mathcal{A} \rightarrow \mathbb{A}(\mathbb{M}(\mathcal{A}))$ defined by

$$
\beta(a)=\{u \in \operatorname{supp}(\mathbb{M}(\mathcal{A})) \mid a \in u\}=(a) .
$$

(ii) Any Stone Markov process $\mathcal{M}=(M, \mathcal{A}, \theta)$ is homeomorphic to $\mathbb{M}(\mathbb{A}(\mathcal{M}))$ via the map $\alpha: \mathcal{M} \rightarrow \mathbb{M}(\mathbb{A}(\mathcal{M}))$ defined by

$$
\alpha(m)=\{a \in \mathbb{A}(\mathcal{M}) \mid m \in a\} .
$$

Proof. (i) The set $\beta(a)$ is the set of good ultrafilters of $\mathcal{A}$ that contain $a$; that is, $\beta(a)=(a)$. By the classical Stone representation theorem, $\mathcal{A}$ and $(\mathcal{A})^{*}$ are isomorphic as Boolean algebras via the map $a \mapsto(a)^{*}$. By the RasiowaSikorski lemma (Theorem 5) and Lemma 14, the good ultrafilters are dense in $(\mid \mathcal{A})^{*}$, therefore $(\mid \mathcal{A})^{*}$ and $(\mathcal{A} \mid)$ are isomorphic as Boolean algebras via the $\operatorname{map}(a)^{*} \mapsto(a)$.

It remains to show that the operations $F_{r}$ are preserved. Let $\mathcal{U}=\operatorname{supp}(\mathbb{M}(\mathcal{A}))$. For each $r \in \mathbb{Q}_{0}$,

$$
\begin{aligned}
\beta\left(F_{r} a\right) & =\left\{u \in \mathcal{U} \mid F_{r} a \in u\right\} \\
& =\{u \in \mathcal{U} \mid \theta(u)(|a|) \geq r\} \\
& =\{u \in \mathcal{U} \mid \theta(u)(\beta(a)) \geq r\} \\
& =F_{r}(\beta(a)) .
\end{aligned}
$$

(ii) The set $\alpha(m)$ is the set of all elements of $\mathbb{A}(\mathcal{M})$ that contain $m$. We first prove that this is a good ultrafilter of $\mathbb{A}(\mathcal{M})$. It is clearly an ultrafilter, as it is represented by a point of a set-theoretic Boolean algebra. To show that it is good, we need to reason that if $a \in \mathcal{A}$ and $F_{r} a \in \alpha(m)$ for all $r<s$, then $F_{s} a \in \alpha(m)$. This follows immediately from the fact that $F_{t} a \in \alpha(m)$ iff $m \in F_{t} a$ iff $\theta(m)(a) \geq t$.

The map $\alpha$ is a a strict embedding, since the two distinguished bases $\mathcal{A}$ of $\mathcal{M}$ and $(\mathcal{A})$ of $\mathbb{M}(\mathbb{A}(\mathcal{M}))$ are isomorphic. This embedding must be a homeomorphism, since $\mathcal{M}$ is saturated. 


\subsection{Duality in Categorical Form}

We present the previous results in a more categorical format. The categories of Aumann algebras (AA) and Stone Markov processes (SMP) were defined in $\S 4$ and $\S 5$, respectively.

We define contravariant functors $\mathbb{A}: \mathbf{S M P} \rightarrow \mathbf{A A}^{\text {op }}$ and $\mathbb{M}: \mathbf{A A} \rightarrow$ $\mathbf{S M P}^{\text {op }}$. The functor $\mathbb{A}$ on an object $\mathcal{M}$ produces the Aumann algebra $\mathbb{A}(\mathcal{M})$ defined in Theorem 22. On arrows $f: \mathcal{M} \rightarrow \mathcal{N}$ we define $\mathbb{A}(f)=$ $f^{-1}: \mathbb{A}(\mathcal{N}) \rightarrow \mathbb{A}(\mathcal{M})$. It is well known that this is a Boolean algebra homomorphism. It is also easy to verify from the definition of morphisms in the category SMP (Definition 13) that it is an Aumann algebra homomorphism.

To see this explicitly, let $A \in \mathcal{A}_{\mathcal{N}}$. We wish to show that

$$
f^{-1}\left(F_{r}^{\mathcal{N}}(A)\right)=F_{r}^{\mathcal{M}}\left(f^{-1}(A)\right) .
$$

Using the fact that

$$
\theta_{\mathcal{N}}(f(m))(A)=\theta_{\mathcal{M}}(m)\left(f^{-1}(A)\right)
$$

we have

$$
\begin{aligned}
m \in f^{-1}\left(F_{r}^{\mathcal{N}}(A)\right) & \Leftrightarrow f(m) \in F_{r}^{\mathcal{N}}(A) \\
& \Leftrightarrow \theta_{\mathcal{N}}(f(m))(A) \geq r \\
& \Leftrightarrow \theta_{\mathcal{M}}(m)\left(f^{-1}(A)\right) \geq r \\
& \Leftrightarrow m \in F_{r}^{\mathcal{M}}\left(f^{-1}(A)\right) .
\end{aligned}
$$

The functor $\mathbb{M}: \mathbf{A A} \rightarrow \mathbf{S M P}^{\text {op }}$ on an object $\mathcal{A}$ gives the Stone-Markov process $\mathbb{M}(\mathcal{A})$ defined in Theorem 20. On morphisms $h: \mathcal{A} \rightarrow \mathcal{B}$, it maps ultrafilters to ultrafilters by $\mathbb{M}(h)=h^{-1}: \mathbb{M}(\mathcal{B}) \rightarrow \mathbb{M}(\mathcal{A})$; that is,

$$
\mathbb{M}(h)(u)=h^{-1}(u)=\left\{A \in \mathcal{A}_{\mathcal{N}} \mid h(A) \in u\right\} .
$$

Another way to view $\mathbb{M}(h)$ is by composition, recalling that an ultrafilter can be identified with a homomorphism $\bar{u}: \mathcal{A} \rightarrow \mathbb{2}$ by $u=\{a \mid \bar{u}(a)=1\}$. In this view,

$$
\mathbb{M}(h)(\bar{u})=\bar{u} \circ h,
$$

where $\circ$ denotes function composition. 
We know from classical Stone duality that this is continuous. We need to verify that it is a morphism. It suffices to verify it on sets of the form (a) as these generate the $\sigma$-algebra. Because $h$ is a homomorphism, we calculate as follows:

$$
\begin{aligned}
\left.\theta_{\mathcal{B}}(u)\left(\mathbb{M}(h)^{-1}(|a|)\right)\right) & =\sup \left\{r \mid F_{r}(h(a)) \in u\right\} \\
& =\sup \left\{r \mid h\left(F_{r}(a)\right) \in u\right\} \\
& =\left\{r \mid \bar{u}\left(h\left(F_{r}(a)\right)\right)=1\right\} \\
& =\left\{r \mid F_{r}(a) \in \mathbb{M}(h)(u)\right\} \\
& =\theta_{\mathcal{A}}(\mathbb{M}(h)(u)((\mid a))) .
\end{aligned}
$$

Theorem 24. The functors $\mathbb{M}$ and $\mathbb{A}$ define a dual equivalence of categories.

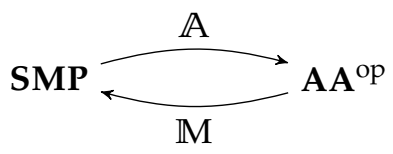

Proof. We need to show that we have a contravariant adjunction $\mathbb{A} \dashv \mathbb{M}$ and that the unit and counit of the adjunction are isomorphisms.

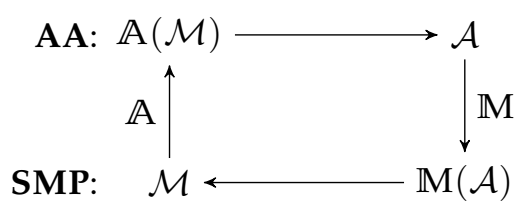

The unit and counit are the natural transformations

$$
\alpha^{\prime}: \mathbb{M} \circ \mathbb{A} \rightarrow \mathbb{I} \quad \beta^{\prime}: \mathbb{A} \circ \mathbb{M} \rightarrow \mathbb{I}
$$

respectively, with components

$$
\alpha_{\mathcal{M}}^{\prime}: \mathbb{M}(\mathbb{A}(\mathcal{M})) \rightarrow \mathcal{M} \quad \beta_{\mathcal{A}}^{\prime}: \mathbb{A}(\mathbb{M}(\mathcal{A})) \rightarrow \mathcal{A},
$$

the inverses of the $\alpha$ and $\beta$ defined in Theorem 23. Here we are using ' to denote functional inverse so as not to conflict with our previous usage of ${ }^{-1}$ as $f^{-1}(X)=\{x \mid f(x) \in X\}$. We have already shown that these are isomorphisms.

To verify that we have an adjunction with unit $\alpha^{\prime}$ and counit $\beta^{\prime}$, it suffices to verify the conditions

$$
\beta^{\prime} \mathbb{A} \circ \mathbb{A} \alpha^{\prime}=\mathbb{I} \quad \mathbb{M} \beta^{\prime} \circ \alpha^{\prime} \mathbb{M}=\mathbb{I} .
$$


Since $\alpha^{\prime}$ and $\beta^{\prime}$ are invertible, these are equivalent to

$$
\mathbb{A} \alpha^{\prime}=\beta \mathbb{A} \quad \mathbb{M} \beta^{\prime}=\alpha \mathbb{M} .
$$

Specializing at components $\mathcal{A}$ and $\mathcal{M}$ and using the definition of $\mathbb{A}$ and $\mathbb{M}$ on morphisms, it suffices to show

$$
\alpha_{\mathcal{M}}^{\prime-1}=\beta_{\mathbb{A}(\mathcal{M})} \quad \beta_{\mathcal{A}}^{\prime-1}=\alpha_{\mathbb{M}(\mathcal{A})} .
$$

For the left-hand equation, as $\alpha_{\mathcal{M}}$ is invertible, all elements of $\mathbb{M}(\mathbb{A}(\mathcal{M}))$ are of the form $\alpha_{\mathcal{M}}(m)$ for some $m \in \mathcal{M}$, and $\alpha_{\mathcal{M}}^{\prime}\left(\alpha_{\mathcal{M}}(m)\right)=m$. Thus for $C \in \mathbb{A}(\mathbb{M}(\mathbb{A}(\mathcal{M})))$,

$$
\begin{aligned}
\alpha_{\mathcal{M}}^{\prime-1}(C) & =\left\{\alpha_{\mathcal{M}}(m) \mid \alpha_{\mathcal{M}}^{\prime}\left(\alpha_{\mathcal{M}}(m)\right) \in C\right\} \\
& =\left\{\alpha_{\mathcal{M}}(m) \mid m \in C\right\} \\
& =\left\{\alpha_{\mathcal{M}}(m) \mid C \in \alpha_{\mathcal{M}}(m)\right\} \\
& =\left\{\alpha_{\mathcal{M}}(m) \mid \alpha_{\mathcal{M}}(m) \in \beta_{\mathbb{A}(\mathcal{M})}(C)\right\} \\
& =\beta_{\mathbb{A}(\mathcal{M})}(C) .
\end{aligned}
$$

The argument for the right-hand equation is symmetric.

\section{Related Work}

Stone duality in semantics originates with the pioneering work of Plotkin [4] and Smyth [5], who discovered a Stone-type duality between the predicatetransformer semantics of Dijkstra and state-transformer semantics. Kozen [6] developed a probabilistic analogue of this duality. Abramsky [7] studied dualities in domain and concurrency theory.

The theory of Stone-type dualities for transition systems has been investigated at length by Bonsangue and Kurz [8] and there have been many recent investigations of Stone-type dualities from the viewpoint of coalgebra and automata theory [30-32]. Recent very interesting work by Jacobs [33] has explored convex dualities for probability and quantum mechanics.

Duality theory for LMPs was discussed by Mislove et al. [10] which is based on Gelfand duality for $C^{*}$-algebras. This is very interesting work, but in rather a different direction from the present work, which is very much in the spirit of logics for Markov processes and is related to bisimulation and its logical characterization [12]. By contrast, the work of Mislove et al. [10] is related to testing. 
The most closely related work to ours is the work of Goldblatt [14] on the role of the Baire category theorem in completeness proofs, and even more closely his work on deduction systems for coalgebras [17]. The main difference between his work and ours is that we have eliminated some of the infinitary axioms that he uses, although we still retain one, and of course we have developed a duality rather than just a completeness theorem. He uses one of his infinitary axioms in order to show countable additivity of the measures that he defines; this is what we have been able to eliminate by our use of the Rasiowa-Sikorski lemma to eliminate the bad ultrafilters. As far as we know this is a new idea.

\section{Conclusions}

As promised, we have proved a duality theorem between Stone-Markov processes and Aumann algebras which subsumes and extends the completeness theorems in the literature. Our treatment improves on the existing axiomatizations as well.

The following novel features appear in our proof:

1. We must remove ultrafilters that fail to satisfy a key infinitary axiom, and we must show that this does not change the represented algebra by showing that these are "rare" in a topological sense (meager).

2. As a result, the usual compactness for Stone spaces fails, and we need a new concept, which we call saturation, instead.

3. We must establish the relevant measure theoretic properties of the Markov kernels that we construct from the algebras. This again uses the Baire category theorem in a crucial way.

4. We define our Markov processes with a distinguished base and use this to constrain the morphisms in order to achieve duality.

There are many variations one can imagine exploring. Perhaps the most interesting one is to consider more general measure spaces and work with different bisimulation notions [34] that apply more generally. Our treatment is not fully localic, and perhaps some of the topological subtleties of the present proof would disappear once we adopted a more localic point of view. 


\section{Acknowledgments}

We would like to thank Nick Bezhanishvili, Marcello Bonsangue, Vincent Danos, Josée Desharnais, Martin Escardo, Rob Goldblatt, Jean GoubaultLarrecq, Clemens Kupke, Alexander Kurz, Franogis Laviolette, Mike Mislove, Dusko Pavlovic, Gordon Plotkin, and Chunlai Zhou for helpful discussions.

\section{References}

[1] M. H. Stone, "The theory of representations for boolean algebras," Trans. Amer. Math. Soc., vol. 40, pp. 37-111, 1936.

[2] P. Johnstone, Stone Spaces, ser. Cambridge Studies in Advanced Mathematics. Cambridge University Press, 1982, vol. 3.

[3] B. Jonsson and A. Tarski, "Boolean algebras with operators I," American Journal of Mathematics, vol. 73, pp. 891-939, 1951.

[4] G. D. Plotkin, "Lecture notes on domain theory," 1983, available from Plotkin's web page as The Pisa Notes.

[5] M. Smyth, "Powerdomains and predicate transformers," in Proceedings of the International Colloquium On Automata Languages And Programming, J. Diaz, Ed. Springer-Verlag, 1983, pp. 662-676, lecture Notes In Computer Science 154.

[6] D. Kozen, "A probabilistic PDL," Journal of Computer and Systems Sciences, vol. 30, no. 2, pp. 162-178, 1985.

[7] S. Abramsky, "Domain theory in logical form," Annals of Pure and Applied Logic, vol. 51, pp. 1-77, 1991.

[8] M. M. Bonsangue and A. Kurz, "Duality for logics of transition systems." in FoSSaCS, 2005, pp. 455-469.

[9] A. Silva, "Kleene coalgebra," Ph.D. dissertation, University of Nijmegen, 2010.

[10] M. Mislove, J. Ouaknine, D. Pavlovic, and J. Worrell, "Duality for labelled Markov processes," in Foundations of Software Science and Computation Structures, FOSSACS, ser. Lecture Notes In Computer Science, I. Walukiewicz, Ed., vol. 2987, 2004, pp. 393-407. 
[11] J. Desharnais, A. Edalat, and P. Panangaden, "A logical characterization of bisimulation for labelled Markov processes," in proceedings of the 13th IEEE Symposium On Logic In Computer Science, Indianapolis. IEEE Press, June 1998, pp. 478-489.

[12] — - "Bisimulation for labeled Markov processes," Information and Computation, vol. 179, no. 2, pp. 163-193, Dec 2002.

[13] L. Cardelli, K. G. Larsen, and R. Mardare, "Continuous markovian logic - from complete axiomatization to the metric space of formulas," in CSL, 2011, pp. 144-158.

[14] R. Goldblatt, "On the role of the Baire category theorem in the foundations of logic," Journal of Symbolic logic, pp. 412-422, 1985.

[15] C. Zhou, "A complete deductive system for probability logic with application to Harsanyi type spaces," Ph.D. dissertation, Indiana University, 2007.

[16] H. Rasiowa and R. Sikorski, "A proof of the completeness theorem of gödel," Fund. Math, vol. 37, pp. 193-200, 1950.

[17] R. Goldblatt, "Deduction systems for coalgebras over measurable spaces," Journal of Logic and Computation, vol. 20, no. 5, pp. 1069-1100, 2010.

[18] P. Billingsley, Probability and Measure. Wiley-Interscience, 1995.

[19] R. M. Dudley, Real Analysis and Probability. Wadsworth and Brookes/Cole, 1989.

[20] P. Panangaden, Labelled Markov Processes. Imperial College Press, 2009.

[21] R. Mardare, L. Cardelli, and K. G. Larsen, "Continuous markovian logics - axiomatization and quantified metatheory," Logical Methods in Computer Science, vol. 8, no. 4, 2012.

[22] C. Zhou. and M. Ying, "Approximation of markov processes through filtration," Theoretical Computer Science, p. in press, Jan 2012.

[23] E.-E. Doberkat, Stochastic Relations. Foundations for Markov Transition Systems. New York: Chapman and Hall, 2007. 
[24] R. Aumann, "Interactive epistemology I: knowledge," International Journal of Game Theory, vol. 28, pp. 263-300, 1999.

[25] — , "Interactive epistemology II: probability," International Journal of Game Theory, vol. 28, pp. 301-314, 1999.

[26] K. G. Larsen and A. Skou, "Bisimulation through probablistic testing," Information and Computation, vol. 94, pp. 1-28, 1991.

[27] A. Heifetz and P. Mongin, "Probability logic for type spaces," Games and Economic Behavior, vol. 35, no. 1-2, pp. 31-53, April 2001.

[28] R. Fagin and J. Y. Halpern, "Reasoning about knowledge and probability," Journal of the ACM, vol. 41, no. 2, pp. 340-367, 1994.

[29] C. C. Chang and H. J. Keisler, Model Theory. North-Holland, 1973.

[30] F. Bonchi, M. Bonsangue, J. Rutten, and A. Silva, “Brzozowski's algorithm (co)algebraically," in Logics and Program Semantics: Essays Dedicated to Dexter Kozen, ser. Lecture Notes In Computer Science, R. Constable and A. Silva, Eds., vol. 7230. Springer-Verlag, 2012, pp. 12-23.

[31] N. Bezhanishvili, C. Kupke, and P. Panangaden, "Minimization via duality," in Logic, Language, Information and Computation - 19th International Workshop, WoLLIC 2012, Buenos Aires, Argentina, September 3-6, 2012. Proceedings, ser. Lecture Notes in Computer Science, vol. 7456. Springer, 2012, pp. 191-205.

[32] M. Gehrke, S. Grigorieff, and J.-E. Pin, "Duality and equational theory of regular languages," in ICALP (2), 2008, pp. 246-257.

[33] B. Jacobs, "Probabilities, distribution monads, and convex categories," Theor. Comput. Sci., vol. 412, no. 28, pp. 3323-3336, 2011.

[34] V. Danos, J. Desharnais, F. Laviolette, and P. Panangaden, "Bisimulation and cocongruence for probabilistic systems," Information and Computation, vol. 204, no. 4, pp. 503-523, 2006. 